\title{
Energy Consumption Evaluation of ZBLE, AOMDV and AODV Routing Protocols in Mobile Ad-hoc Networks
}

\author{
Rani Sahu ${ }^{1, *}$, Sanjay Sharma1, Murtaza Abbas Rizvi² \\ ${ }^{1}$ Department of Computer Applications, Maulana Azad National Institute of Technology, Bhopal, India \\ ${ }^{2}$ Department of Computer Technologies and Application, National Institute of Technical Teachers Training and Research (NITTTR), Bhopal, \\ India
}

\author{
Email address: \\ rani.princy28@gmail.com (R. Sahu) \\ ${ }^{*}$ Corresponding author
}

\section{To cite this article:}

Rani Sahu, Sanjay Sharma, Murtaza Abbas Rizvi. Energy Consumption Evaluation of ZBLE, AOMDV and AODV Routing Protocols in Mobile Ad-hoc Networks. Advances in Wireless Communications and Networks. Vol. 5, No. 2, 2019, pp. 41-51.

doi: 10.11648/j.awcn.20190502.11

Received: July 23, 2019; Accepted: August 15, 2019; Published: September 25, 2019

\begin{abstract}
Background / Objective: Mobile ad hoc network (MANET) creates a transient network that does not depend on any infrastructure or central administration mechanism. Such networks are made up of wireless mobile nodes. These nodes have to rely on batteries due to lack of stable power supply, so energy consumption is considered one of the main difficulties of this type of network. These nodes change their positions immediately, so the battery is depleted very suddenly, which affects network life. The best routing can be achieved by improving network quality, keeping high energy in mind by multipath routing in ad hoc networks using zone-based technology. The main objective of this research is to evaluate some popular performance metrics with energy efficiency. In this research, the zone-based leader Election Energy-Constructed AOMDV Routing Protocol (ZBLE) has been compared to the traditional routing protocols AODV and AOMDV, suggesting that the ZBLE protocol exhibits better results. Methods / Statistical analysis: In this paper, the routing protocol ZBLE based on zonebased technique is used. In this, the leader node is selected using the node's energy label, power analysis, and node position tracking function. The best route has been achieved by the leader node selection algorithm and power analysis. Conclusion: Most energy-related protocols are based on single-route routing, which has not been able to pay much attention to the accumulation of energy, and there have been attempts to make multi-way routing protocols also energy-based protocols, but also these protocols have been unable to make good use of energy. Through the NS2 simulator, network life is extended by reducing energy consumption by using a new energy-based multi-way routing protocol ZBLE. The results of this routing protocol performance evaluation suggest that the ZBLE protocol is superior to the AOMDV and AODV protocols. It is a zonebased technique that works by concentrating on the highest energy of nodes. Simulation results reported that the performance of ZBLE outperformed that of AOMDV and AODV. Applications / improvements: Zone-based technology can be easily used for extremely large networks. Ad-hoc network performance has been improved by improving network quality using this protocol.
\end{abstract}

Keywords: Energy Efficient Routing Protocol, MANETs, Energy Efficiency, Multipath Routing Protocol, Zone Based Routing Performance

\section{Introduction}

Different wireless mobile nodes collectively form a network that does not depend upon any central administration system. This kind of community is known as Mobile Adhoc Network (MANET). [1, 2]

With the continuous advancement of communication technologies, the dependence of humans on wireless mobile gadgets is growing and the usage of its applications in the coming days could be popular and protected. Various dilemmas were derived from the ad hoc network, including bandwidth optimization, transmission quality enhancement, and power control.

To do away with those challenges in the present, the 
Internet Engineering Task Force has proposed several procedures and schemes on certain techniques. All the possible efforts are being made to overcome this problem in several educational and technical projects [3].

The fundamental purpose of MANETs is to make system communication powerful and effective so that the quality of the network can be ensured. All the devices existing in MANET are dependent on the battery. Due to the restricted battery of mobile gadgets, the network's life and connectivity decreases which affect the network [4]. That's why energy consumption in MANETs has been significant trouble since the past few days. The nodes in MANET are dependent on the battery that is why energy consumption has come to be a major dilemma. In today's development, wireless networks (WNs) are being used as extremely energetic in every field. They had been utilized in various applications, but some of the vulnerabilities, inclusive of mobility, diversity, lack of resources and so on, finding an optimum route are very complicated and problematical. Due to the mobility of the nodes in the network, the nodes quickly change their position, inflicting the battery to die very quickly, thereby reducing the existence of such networks. Therefore, to growth the network lifecycle, there may be a need to boom the battery life of the node. By reducing the energy consumption on a particular node, the quality of the network can be ensured with the help of the extension of the node's battery existence. In order to solve this dilemma, we need a routing protocol that may select the route based on the analysis of the energy and power of the node in Zone-Based Technology. Various Energy-Aware routing protocols had been evolved for the purpose of diminishing energy consumption in MANET [5-9]. Researchers have concentrated on the design of such protocols that preserve the energy so that the network service can be assured for as long as feasible [10-12].

During the last several years, certain routing protocols have been developed to enhance the life of the network, including a multipath route protocol. Due to the already available backup route in the multipath route protocol, the problem of the route failed. Several algorithms had been developed for multipath routing with energy efficiency performance. With the improvement of network density in multipath routing, a further value of production will increase considerably; this is the main trouble of multipath routing.

The problem has not been solved by the existing energyaware routing protocols in the ad hoc network. This problem may be rid of using zone-based technology. Zone-based technology is geographically a virtual organization of networks in close groups of nodes. Several zone techniques have been proposed in the mobile ad hoc network in literature [13-18]. The main problem of these techniques is the energy consumption in their zone-based structure even as changing the network structure.

As mentioned in advance, every node present in the Adhoc network eliminates its energy over the years because of a restricted battery and a limited energy supply, which impacts network connectivity. Some major nodes along with Zone's
Leader nodes are precious due to the fact they are more appropriate for packet transmission due to high energy than other nodes. A reliable routing protocol with the zone-based scheme will mainly preserve its structure by conserving the energy efficiency of the Zone leader nodes. The main purpose of this paper is to demonstrate against the performance evaluation of the protocol based on the Zonebased technology, with the routing protocol, AOMDV, and AODV. This research work provides a standard for future study of the development of a zone-based routing protocol, which is more efficient in terms of energy consumption and excessive network lifetime.

\section{Routing Protocols of MANETs}

Different routing protocols are available in MANET for improving your current traffic load and the use of battery power well [19]. Any protocol has a big impact on the performance of the network, so selecting the right protocol is a very critical decision [39]. Protocols found in MANET can be categorized in specific methods relying on different theories. Based on the routing strategy, the routing protocol may be categorized into three types: (i) Proactive (ii) Reactive (iii) Hybrid. [20-23]

Proactive or table-driven routing protocol maintains the routing table of every node. Distribute the statistics associated with information about each node present in the network, through the same routing table, across the whole network. Examples of active routing protocols are DSDV, WRP, CGSR, and OLSR. The reactive or demand path protocol path is established only when any nodes need to communicate with each other. Some examples of this are AdHoc On-Demand Distance Vector (AODV), Dynamic Source Routing (DSR).

The Hybrid Routing Protocol is developed with the functions of both the reactive and proactive protocols. Examples of hybrid routing protocols are ZRP, ZHLS.

Traffic volumes associated with the demand for traffic are one of the main troubles of this type of protocol. Some zone routing protocols come in the hybrid routing protocol.

\section{1. $A O D V$}

The mobile Ad Hoc network uses the on-demand approach to find routes for every kind of Network and the responsive path protocol performs an important role. The source node in the AODV protocol only establishes and maintains the route as long as the data packet needs to be transmitted. Information about the next-hop route from the routing table to the destination node by each node can be obtained. This protocol uses Route Request (RREQ), Route Reply (RREP) and Route Error (RERR) messages to search and route routing using the Hop-by-Hop routing approach. In addition, Hello messages are used to keep network connectivity. 


\section{2. $A O M D V$}

A multipath extension of the ad-hoc on-demand distance vector (AODV) routing protocol is the Ad-Hoc on-demand multipath distance vector (AOMDV) routing protocol. Hopto-hop routing concept and distance vector idea concept is used to discover on-demand routes. This protocol plays an important role in maintaining the characteristics of loopfreedom and disjoint. Intermodal coordination removes overheads and provides better performance than other protocols. Along with this, there are also available alternate routes already available, so that another route can be used if one route fails.

\section{3. $Z B L E$}

The main purpose of this protocol is to help maximize network life time by reducing the energy consumption of the wireless network nodes through zone-based technology. This protocol is developed by evaluating the AOMDV protocol only. In order to establish communication in any nodes, optimum path is selected from multipath by adding high energy and power nodes so that the data can be successfully transmitted from source to non-delayed destination. Through this protocol, the entire network can be divided into several areas based on network decomposition. The main purpose of this protocol is to keep energy consumption in mind so that we can try to improve the performance of the life of the network. The main function of this protocol is to select the best energy route by obtaining energy, power and node tracking [24].

\section{Literature Review}

In this paper [25], the author proposed a modified energy constraint (MEC-AOMDV) protocol for an on-demand routing protocol based on AOMDV for the Mobile Ad Hoc network. Proposed MEC-AOMDV to connect the load balancing and transmission power control approach can be used so as to reduce the energy consumption to extend the life of the network. Good results in all network sizes are displayed in terms of performance metrics such as throughput, packet distribution ratio and dropped packets. MEC-AOMDV maximizes the lifetime of the network by reducing the total energy consumption for all traffic conditions compared to the original AOMDV.

In this paper [26], a new multipath routing protocol, AOMR-LM was proposed to balance the consumption energy by protecting the energy-aware routing and the residual energy of the nodes in the mobile Adhoc network. Using the energy-aware mechanism, it was developed by revising the existing multipath routing protocol AOMDV. The lifetime of the network the concept can be improved energy consumption. To find the appropriate values, the coefficient $\alpha$ is analyzed, which is necessary to preserve the node residual energy and define the class of a node during the post-forwarding process. It has been compared with AOMV and JD-AOMDV protocol which increases the lifespan and is able to balance consumption energy.

In this paper [27], the Author has proposed a new routing protocol, which uses the cost function. It considers both residual battery power and current traffic load node. The comparison of the results with the existing protocol is done using Network Simulator 2.35 and it has been found that the proposed EOMVV performs better in the high packet distribution part and high energy efficiency period. There is also end-to-end delay and lower routing overhead. This work can be carried out using other types of mobility models and different traffic models.

In this paper [28], Author has proposed an energy-aware AOMDV (EA-AOMDV) routing protocol. Considering both the residual energy and the minimum residual energy of a node, the paths with weak paths are avoided. The proposed EA-AOMDV protocol shows better performance in life time, average consumption energy, end-to-end delay, normalized route load, packet distribution ratio and distributed packets.

In this paper [24], the author has proposed ZBLE (Zonebased Leader Election Energy-Created AOMDV Routing Protocol) based on Zone Technology. This protocol has been developed by modifying AOMDV. Zone Leader nodes and zone members have been selected using the value of energy, position, and power of the node. Using better results of the quality of service parameters such as packet distribution ratio, energy consumption, network life, and throughput, using NS-2.35 has proved that the proposed ZBLE protocol has given better results than modern two protocols AODV and AOMDV.

In this paper [38], the author has considered delay in MANET and reducing energy consumption. Important factors like residual energy, node connectivity, and choice of cluster heads have been included to calculate the available bandwidth. Creating cluster routing algorithms using the smallest path multicast tree, cluster members as group leaders and select cluster members as leaf nodes are very easy to choose from. By refining some existing protocols, the proposed approach has been displayed.

\section{Research Methodology}

Evaluation of the overall performance of ZBLE, AOMDV and AODV routing protocols in this research especially based on energy consumption. The performance has been evaluated by way of simulating those protocols via NS-2 Version ns2.35 (Network Simulator - ns2) [20-31]. The simulation workflow is shown in figure 1 .

Due to the excessive cost and shortage of flexibility in MANET, simulation is done in the field of research with the help of simulation equipment. Glomosim, qulanate, ns2 and opnet are a few of the current simulation tools. These simulation tools can be used to evaluate the capability of any protocols. Here we have used the NS2 simulation tool. This simulator was developed at the University of California at Berkeley.

NS-2 discrete event simulators can be used to simulate different kinds of internet communication. It is made the 
usage of languages like $\mathrm{C}++$ and TCL. The TCL interpreter is used for the user. That means the TCL script for the network is written through the user. The result of the simulation is to determine the different metrics the usage of the trace file. Network animator is used to visualizing the simulation that comes with the package.

In this research, the performance evaluation of the selected routing protocol, using the event-driven ns2.34 simulator, depends on the simulation of the evaluation 50, 75 and 100 wireless nodes. Simulation has been implemented randomly using the Random Mobility model [34]. Nodes are placed in the setup in the rectangular area of 1507 meters $x 732$ meters in the simulation area for 600 seconds of simulation time. All nodes have been initialized from the initial energy $50 \mathrm{~J}$. Simulation environment setup and configuration is represented through figure 2 .

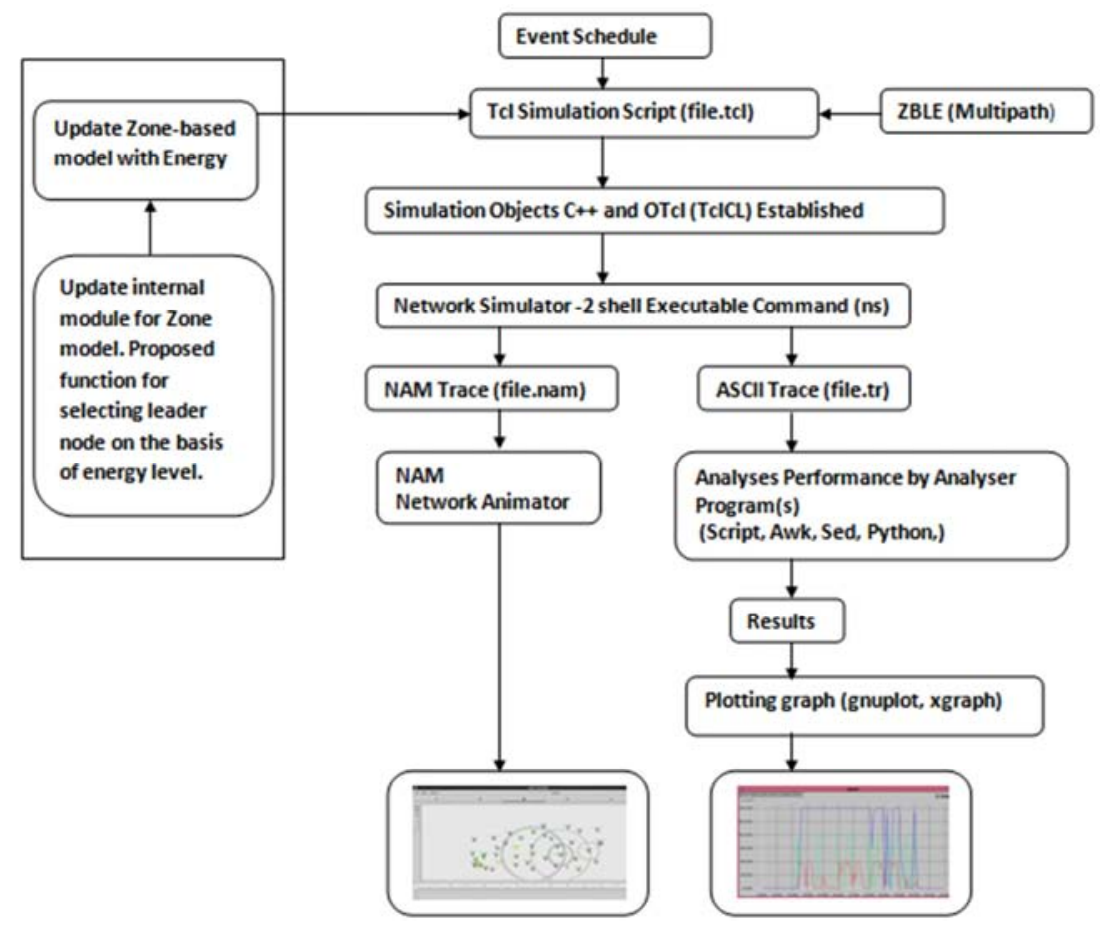

Figure 1. Simulation Workflow.

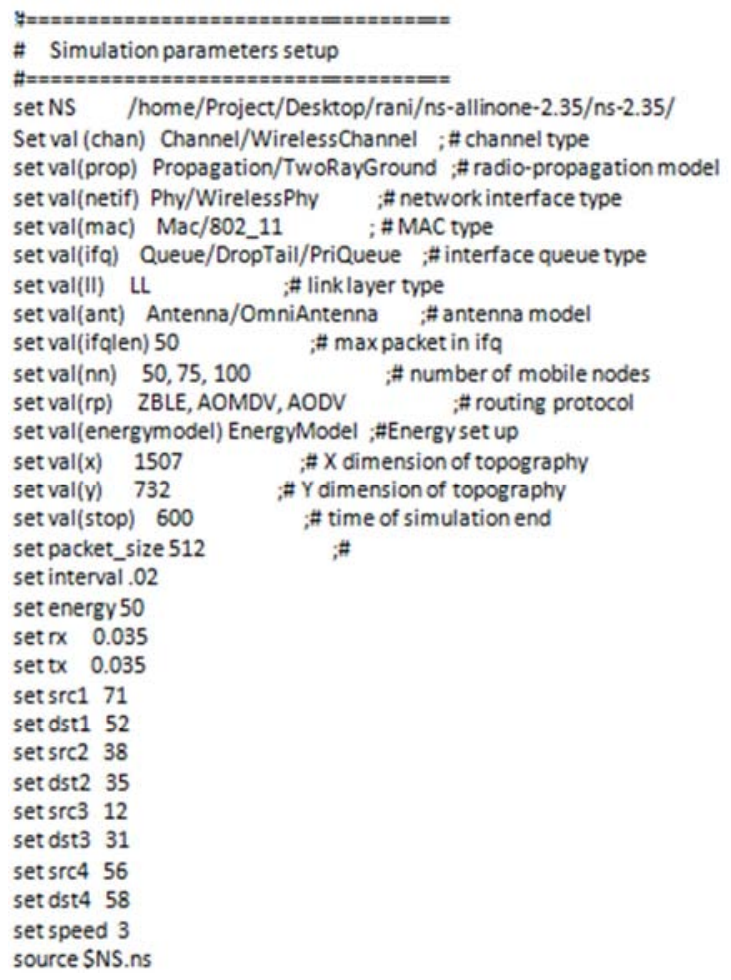

Figure 2. Simulation Environment Configuration. 
Traffic has been generated using a continuous bit rate (CBR). Packet size has been kept to send 512 bytes and 4.0 packets / second rates. Table 1 simulation environment setup and configuration is shown for a fair comparison between all protocols.

Table 1. Simulation Parameters.

\begin{tabular}{ll}
\hline PARAMETER & VALUE \\
\hline Channel Type & Wireless Channel \\
Radio-propagation Model & TwoRayGround \\
MAC type & $802 \_11$ \\
Interface queue type & DropTail / PriQueue \\
link layer type & LL \\
Antenna Model & OmniAntenna \\
Max packet in ifq & 50 \\
Number of mobile nodes & $50,75 \& 100$ \\
Routing protocol & AODV, AOMDV \& ZBLE \\
Energy set up & Energy Model \\
X dimension of topography & $1507 \mathrm{~m}$ \\
Y dimension of topography & $732 \mathrm{~m}$ \\
Time of simulation end & $600 \mathrm{~s}$ \\
Packet size & 512 \\
Initial Energy of node & $50 \mathrm{~J}$ \\
Rx & $0.035 \mathrm{~W}$ \\
Tx & $0.035 \mathrm{~W}$ \\
idlePower & $1.0 \mathrm{~W}$ \\
sleepPower & $0.001 \mathrm{~W}$ \\
Rate & 4 Packet/s \\
Speed & $3 \mathrm{~m} / \mathrm{s}$ \\
\hline &
\end{tabular}

\subsection{Energy Consumption Model}

The network interface of the node reduces energy according to parameters like NIC spectrum, packet size, and used bandwidth. Its detail has been explained with the help of Figure 3. Energy consumption can be measured using the energy model $[32,33]$. On the basis of parameters such as transmission, reception, idle, sleep, transition power and transaction time, the node consumes the energy available (initial energy). This research, the parameters of the energy model used and the specific value is displayed from the help of Table 2 and its detail is displayed through figure 3.

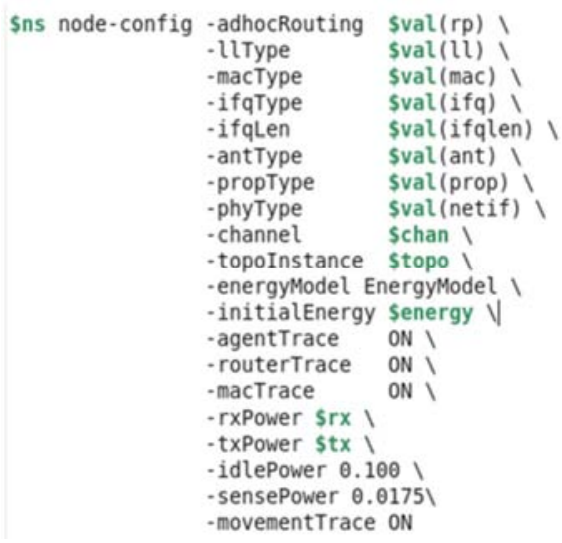

Figure 3. Energy Consumption Model.

Table 2. Energy models values.

\begin{tabular}{llll}
\hline Transmission Power & Receiving Power & Idle Power & Initial Energy (EI) \\
\hline 0.035 watt & 0.035 watt & 0.100 watt & 50 Joules \\
\hline
\end{tabular}

In all protocols, the energy consumption model Carlos et al. [34, 35] has been used. Energy system modeling and energy modeling are used to create and analyze energy-based models in computer systems of energy systems. Energy modeling depends on theoretical energy consumption.

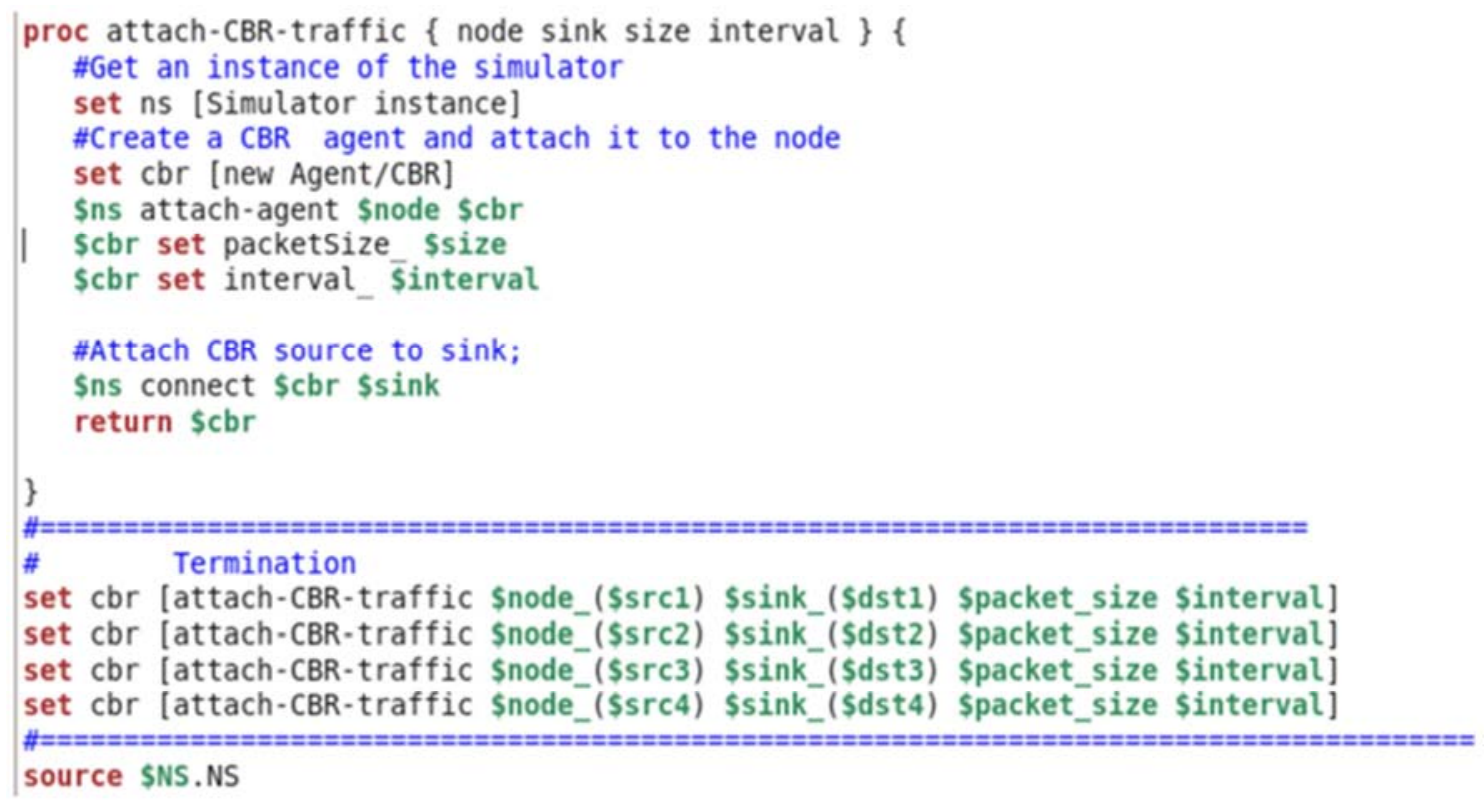




\subsection{Communication Model}

This research uses the continuous bitrate (CBR) source of traffic patterns. All the nodes (the original and the target) in the entire network are randomly spread. The constant bit rate (CBR) traffic is unreliable; there is no guarantee of transmission to data destination as there is no connection establishment stage in it. Also, no acknowledgment is made in this regard. Packet size of 512 bytes has been taken. The communication pattern has been set to 50,75 and 100 nodes. Simulation parameters are presented by Table 7. The communication pattern of 4 connections is shown in Figure 5.

\section{Description of Simulation}

\section{Environment \& Experimental Setup Performance Metrics Used in Simulation}

To evaluate the performance of ZBLE, AOMDV and AODV protocols, use different quantitative indicators like Throughput, End-to-End Delay, Packet Delivery Ratio (PDR), Energy Consumption and Network Lifetime.

Throughput:

It is the ratio of the number of data packets received from the source to the destination, which have successfully reached per unit time through the network on the communication channel.

End To End Delay:

The time taken to send the data packet to the destination node by the source layer is known as the average end-to-end delay.

Packet Delivery Ratio:

This indicates the ratio of packets successfully received on the destination node, in a period, to the data packets sending by the entire source correctly.

\section{Energy Consumption:}

The amount of energy consumed during the simulation time through each node present in the network is indicated by energy consumption.

Network Lifetime:

It refers to the duration of consumption per node to eliminate the battery of the nodes present in the network.

In this section, the results that were generated during the simulation have been included. The trace file has been analyzed using AWK scripting language $[36,37]$ for each scenario.

The output is stored in the matrix by calculating the energy tracking function of the nodes and the energy tracking function of the nodes by taking input through trace files obtained after simulation. The calculation of energy consumed in AWK scripting language is displayed in figure 5 .

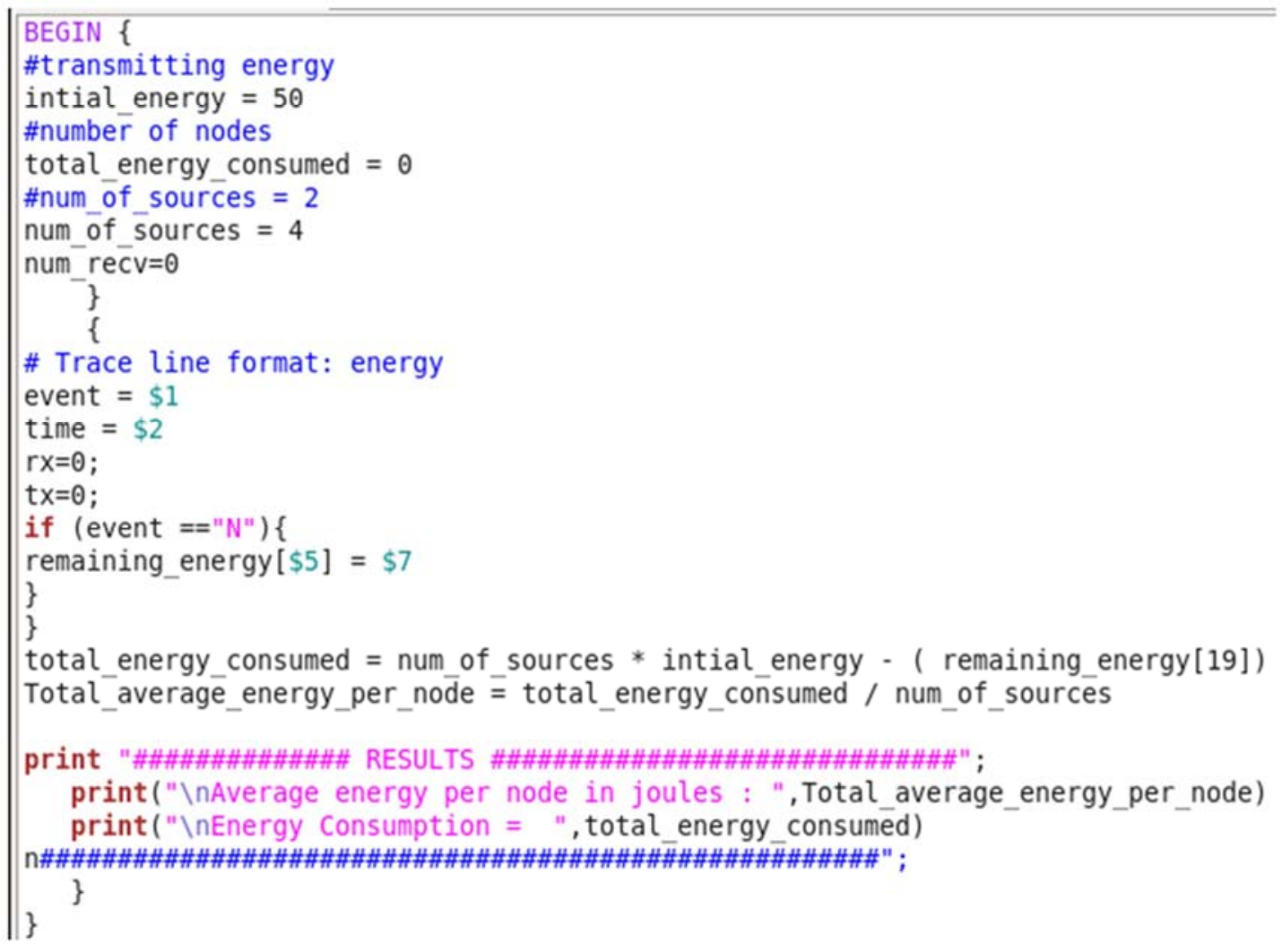

Figure 5. Compute Consumed Energy and Energy Tracking Function.

\subsection{Screenshots of Network Topology}

Screenshots from the network topology of simulation scenario is given in Figure 6. 


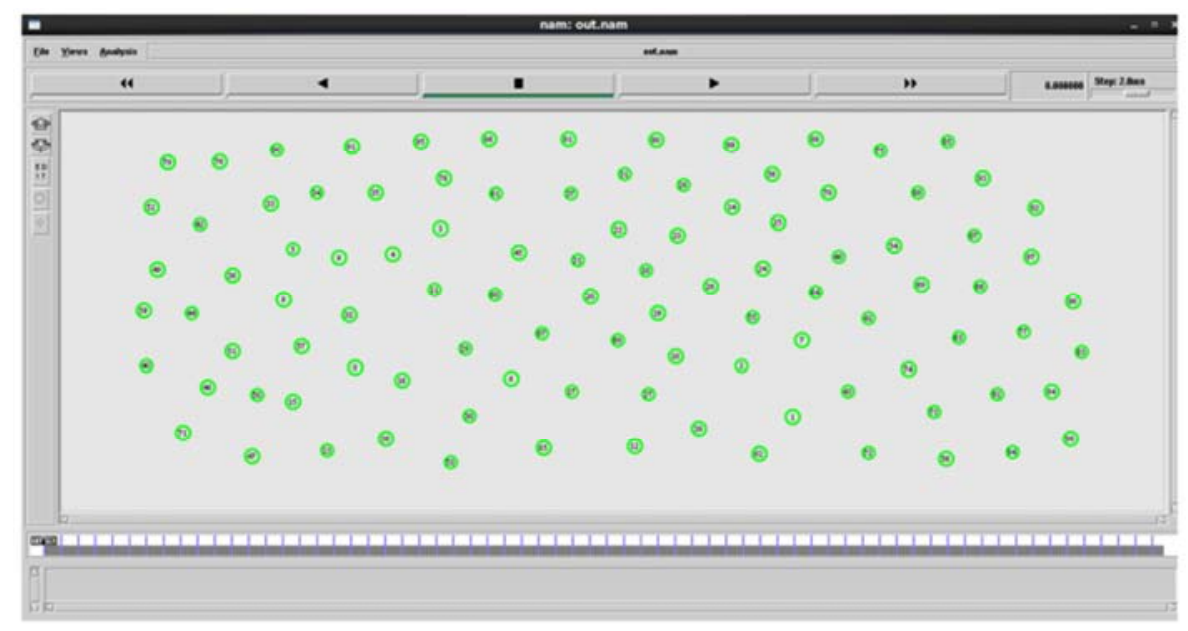

(a) indicates the initial random distribution of the nodes in the area

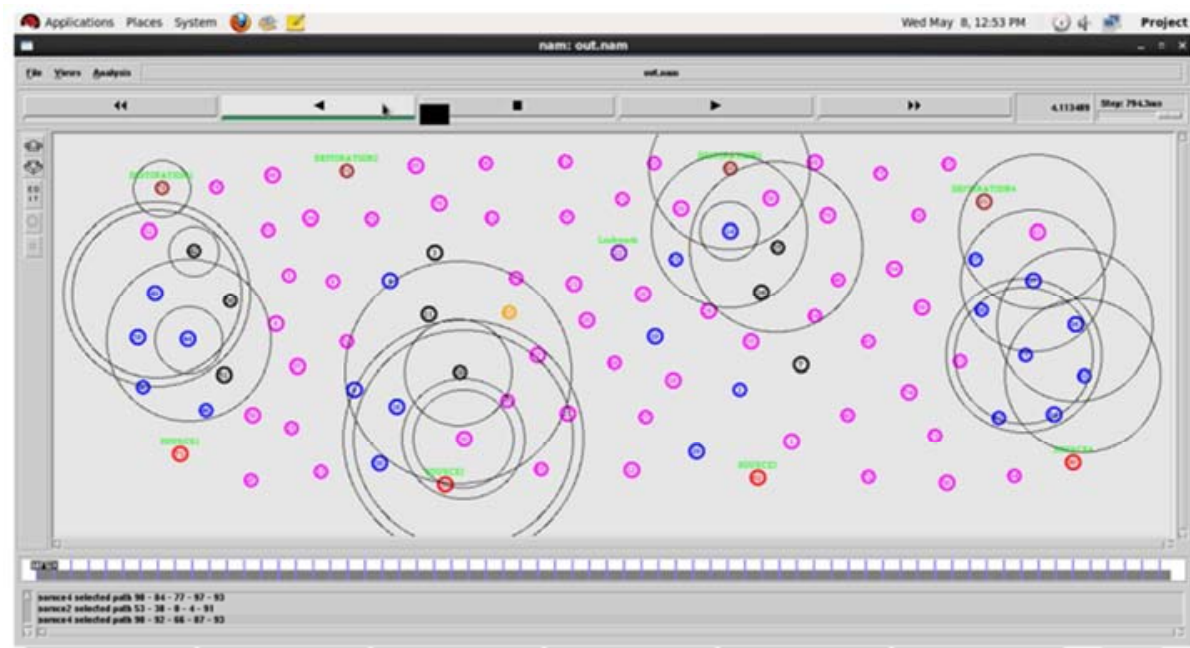

(b) shows the process of ZBLE Protocol

Figure 6. Network Topology Simulation Scenarios.

Results obtained in relation to Throughput, PDR, End to End Delay, Energy Consumption and Network Lifetime in different number of nodes present in the network are displayed in Tables 3, 4, 5, 6 and 7.

Table 3. Throughput comparison.

\begin{tabular}{llll}
\hline \multirow{2}{*}{ Number of Nodes } & \multicolumn{4}{l}{ THROUGHPUT } & \\
\cline { 2 - 4 } & AODV & AOMDV & ZBLE \\
\hline 50 & 6718.66 & 6852.44 & 12666.85 \\
75 & 8708.63 & 8979.59 & 22366.39 \\
100 & 9249.71 & 15186.84 & 26055.05 \\
600 & 11863.74 & 11218.82 & 16920.86 \\
\hline
\end{tabular}

Table 4. Packet Delivery Ratio comparison.

\begin{tabular}{llll}
\hline \multirow{2}{*}{ Number of Nodes } & \multicolumn{3}{l}{ Packet Delivery Ratio } \\
\cline { 2 - 4 } & AODV & AOMDV & ZBLE \\
\hline 50 & 78.9817 & 80.5544 & 87.5352 \\
75 & 51.2045 & 52.7977 & 84.82134 \\
100 & 54.3859 & 84.82134 & 83.4531 \\
\hline
\end{tabular}

Table 5. End to End Delay comparison.

\begin{tabular}{llll}
\hline \multirow{2}{*}{ Number of Nodes } & \multicolumn{4}{l}{ End To End Delay } \\
\cline { 2 - 4 } & AODV & AOMDV & ZBLE \\
\hline 50 & 479.44 & 451.778 & 295.526 \\
75 & 757.079 & 841.936 & 301.801 \\
100 & 907.463 & 962.646 & 395.19 \\
\hline
\end{tabular}

Table 6. Energy Consumption comparison.

\begin{tabular}{llll}
\hline \multirow{2}{*}{ Number of Nodes } & \multicolumn{3}{l}{ Energy Consumption } \\
\cline { 2 - 4 } & AODV & AOMDV & ZBLE \\
\hline 50 & 95.4259 & 95.6652 & 72.7573 \\
75 & 157.634 & 157.991 & 153.871 \\
100 & 196.709 & 196.558 & 173.805 \\
\hline
\end{tabular}

Table 7. Network Life Time comparison.

\begin{tabular}{llll}
\hline \multirow{2}{*}{ Number of Nodes } & \multicolumn{3}{l}{ Network Lifetime } \\
\cline { 2 - 4 } & AODV & AOMDV & ZBLE \\
\hline 50 & 42.2749 & 42.2929 & 52.5456 \\
75 & 42.3658 & 42.0088 & 46.2503 \\
100 & 22.2908 & 22.4415 & 36.5234 \\
\hline
\end{tabular}




\subsection{Throughput}

Figure 7, reflects the variation of the throughput for ZBLE, AOMDV, and AODV. When the number of nodes $(50,75$, 100) increases in the form of nodes, the throughput is increased. Better performance than both AOMDV and AODV in the case of throughput of the ZBLE routing protocol. The distance and stability of the route give the advantage of the ZBLE routing protocol to reduce packet loss and maximize throughput.

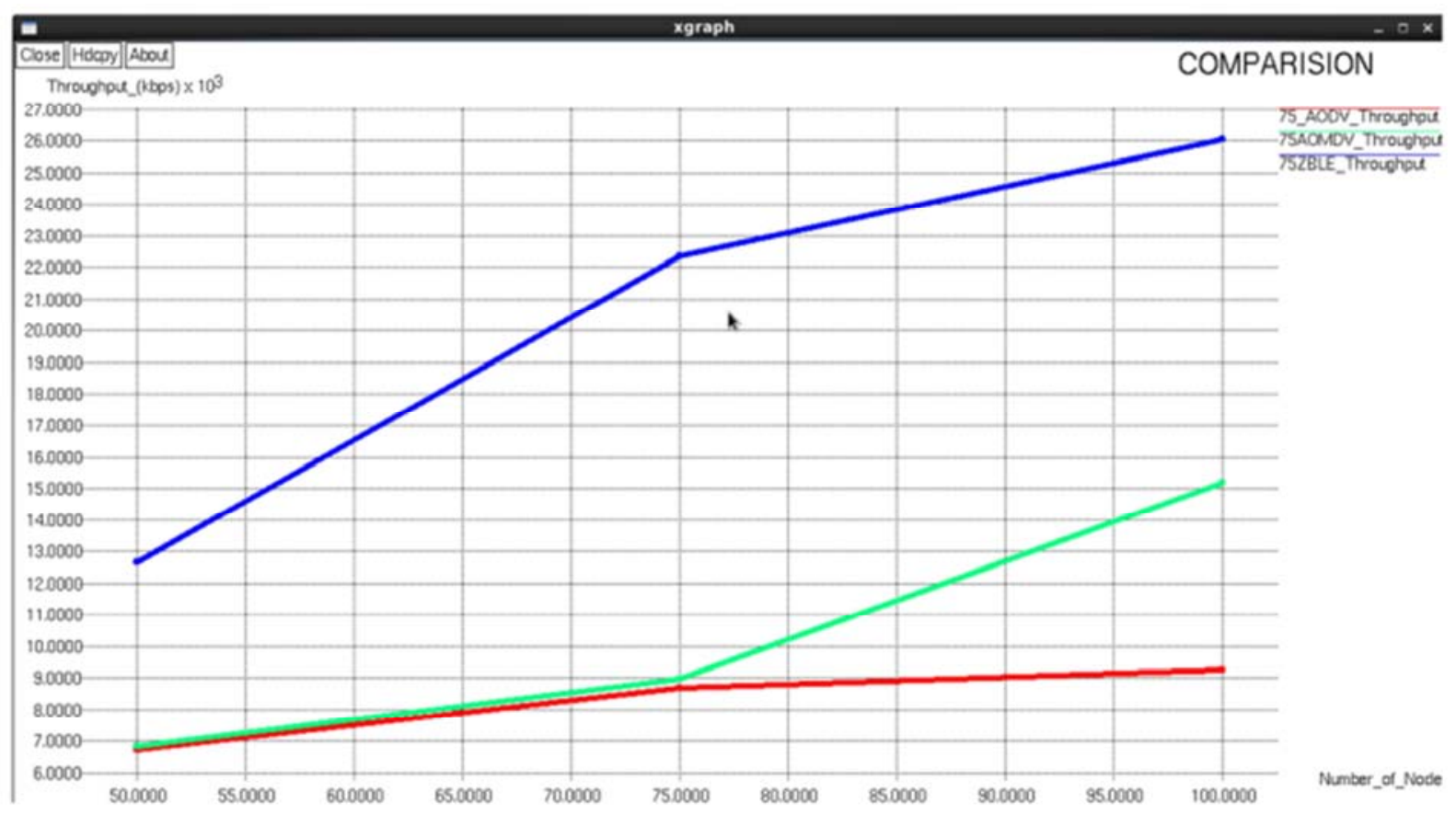

Figure 7. Throughput comparison.

\subsection{End to End Delay}

Figure 8 shows end-to-end delay for ZBLE, AOMDV and AODV, when the number of nodes varies in the form of 50 ,
75 and 100 nodes, when the number of nodes is end-to-end delay Increases with. The ZBLE routing protocol performs better than both AOMDV and AODV.

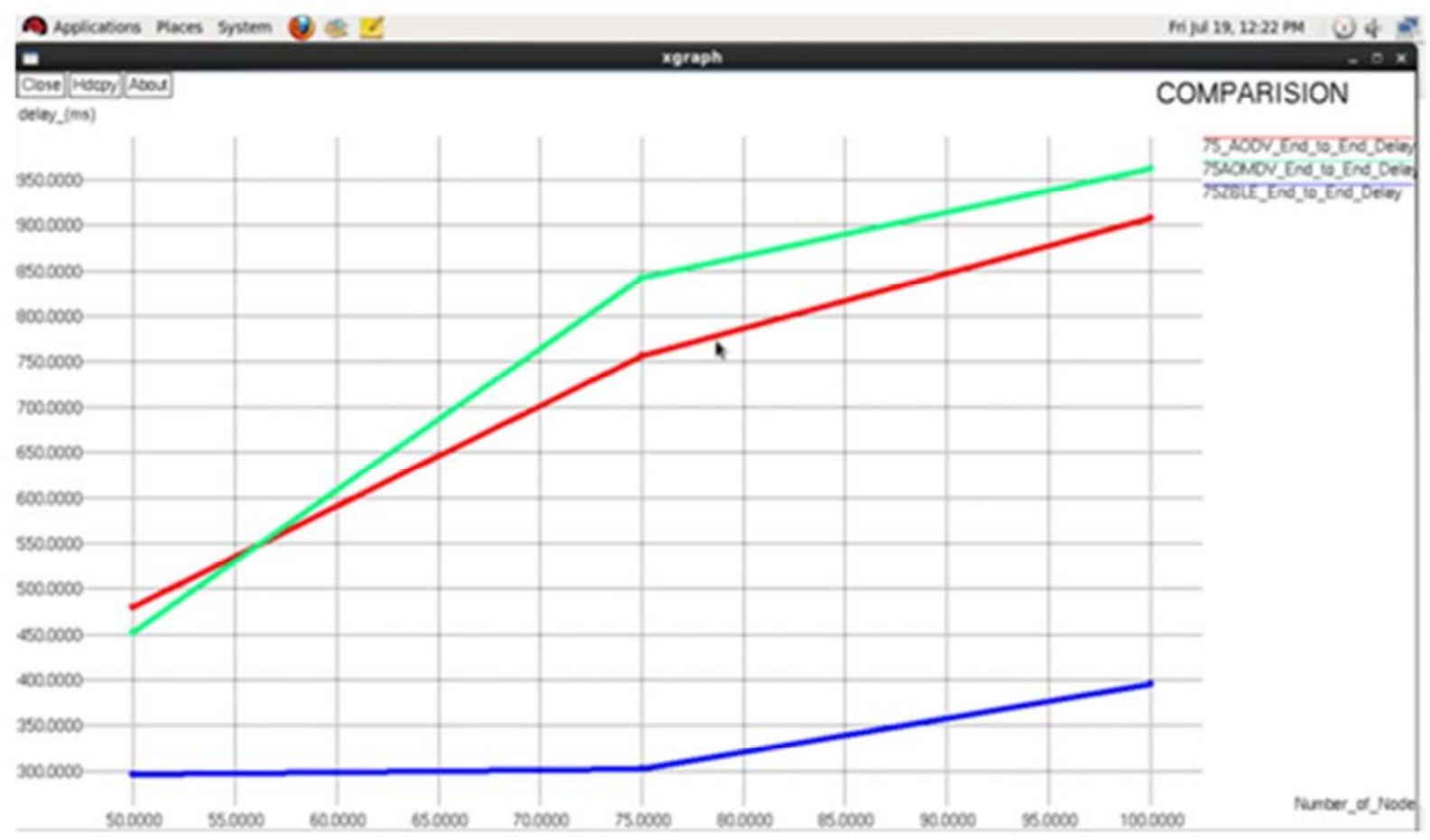

Figure 8. End to End Delay comparisons. 


\subsection{Packet Delivery Ratio}

Figure 9 shows the variation of packet delivery ratio for ZBLE, AOMDV, and AODV. When the node nodes increases as $(50,75,100)$ nodes, the packet delivery ratio decreases.
The ZBLE has higher packet delivery ratio than both AOMDV and AODV. The ZBLE routing protocol selects the most stable route toward the destination. This decreases the possibility of link failure and minimizes packet loss.

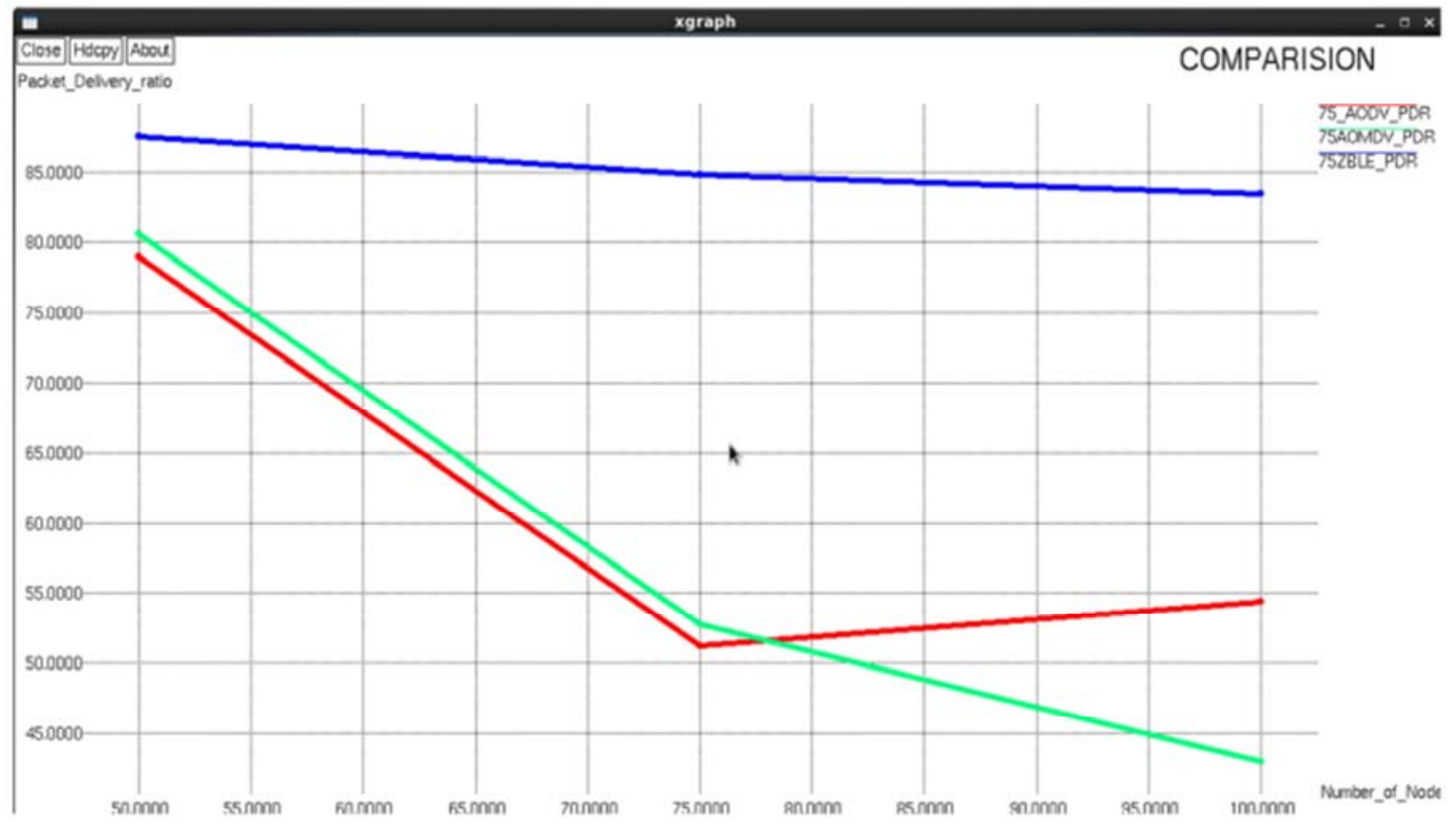

Figure 9. Packet Delivery Ratio comparisons.

\subsection{Energy Consumption}

The amount of energy consumed with the number of nodes from 50, 75, 100 nodes for ZBLE, AOMDV and AODV is shown in Figure 10. Increasing the number of nodes increases energy consumption as well. Compared to both AOMDV and AODV, the ZBLE routing protocol consumes less energy. ZBLE protocol categorizes the routes according to the energy level of the nodes. Along with this, packet distribution and traffic through low level of energy is consumed with low energy consumption. In jab, each area is able to avoid low-energy nodes for data forwarding, so high energy path is selected which reduces energy consumption.

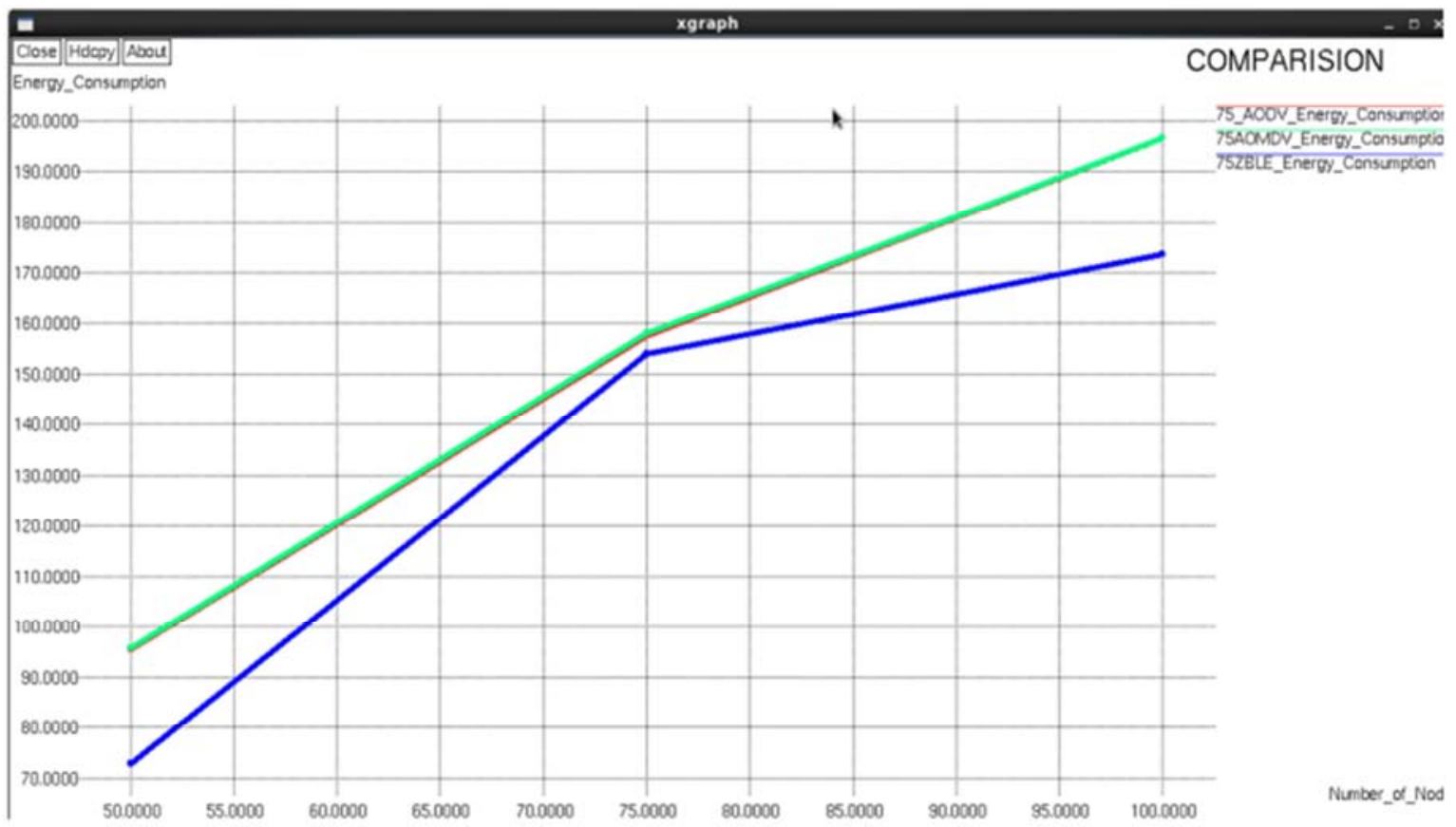

Figure 10. Energy Consumption comparisons. 


\subsection{Network Lifetime}

Network Life Time presents the performance of the three protocols ZBLE, AOMDV, and AODV, along with the growing number of nodes, in Figure 11. Number of nodes is taken 50, 75 and 100.

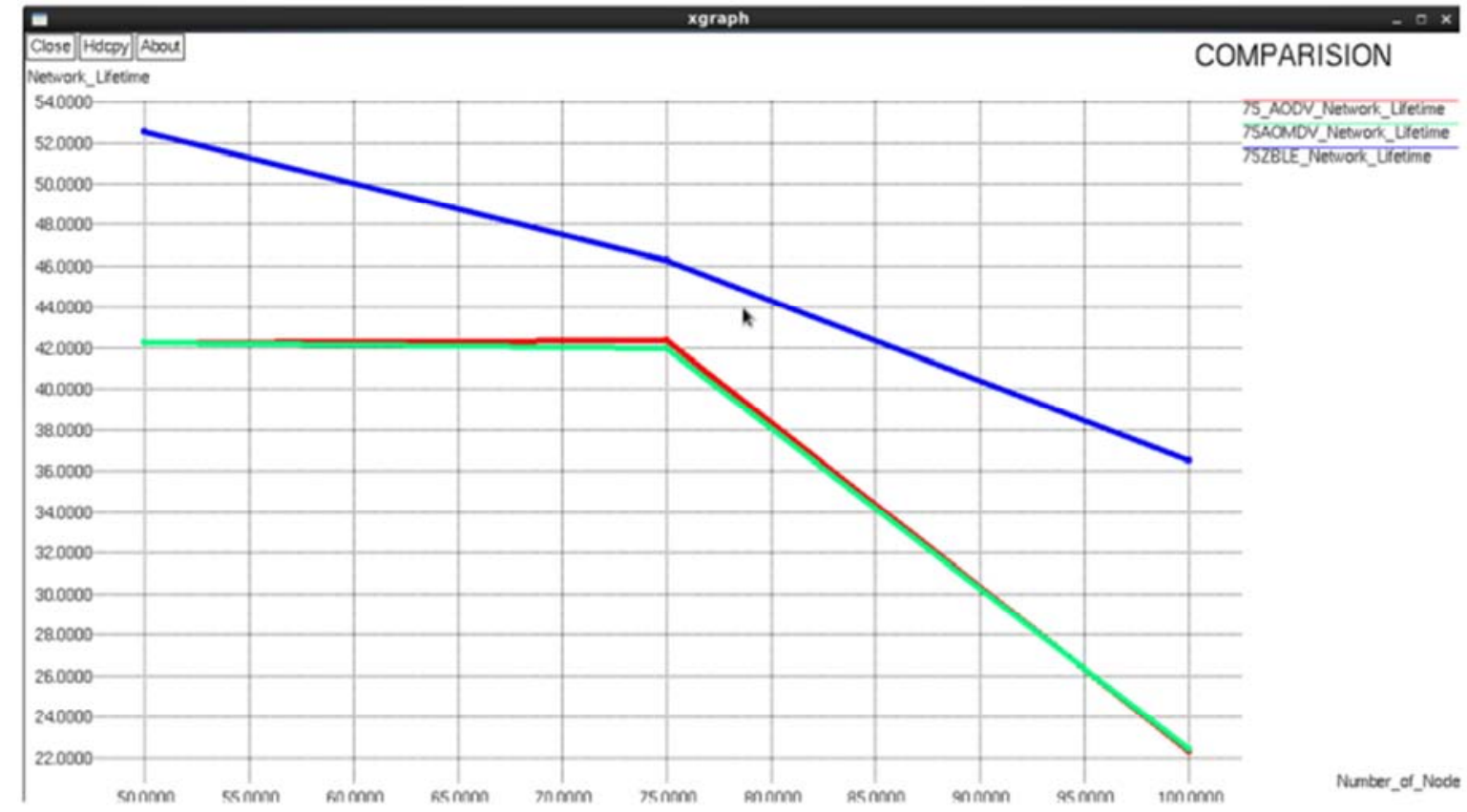

Figure 11. Network Lifetime comparisons.

\section{Conclusion}

In this Work, the performance of Zone-Based Energyefficient Protocol ZBLE has been designed by modifying the Multi-Path Routing Protocol AOMDV to choose the best path in the Zone-based environment in the wireless network. ZBLE enhances the performance of the ad hoc network. The proposed protocol is a multipath route protocol that uses an energy-aware mechanism that uses high-energy nodes with nodes and link connection so that the best-searched path which is having the highest energy is selected for data transfer. After conducting rigorous simulation/experiments to measure the performance of newly design ZBLE protocol it is analyzed that the performance is enhanced in comparison with the wellknown standard protocols such as AOMDV and AODV. Best performance of ZBLE is at Number of nodes 75 and at this value, there is a maximum increase of $61.06 \%$ in throughput as compared to AODV. The average improvement in ZBLE over AODV is $51.70 \%$ and over AOMDV is $45.99 \%$. The results of simulation experiments show that ZBLE performance is better than AODV, and AOMDV also increases the energy consumption and life of the whole network.

\section{References}

[1] Ali, M.; B. G. Stewart; A. Shahrabi; A. Vallavaraj; Multipath routing backbones for load balancing in Mobile Ad hoc Networks, 2012 16th IEEE Mediterranean Electrotechnical Conference, pp. 749-752.
[2] Terdal, Sujatha P.; V. D. Mytri; A. Damodaram; A link quality based dispersity routing algorithm for mobile Ad hoc networks, 2012 International Journal of Computer Network and Information Security 4.9.

[3] Zeng; Qing-An; Dharma P. Agrawal; Handoff in wireless mobile networks, 2002 Handbook of wireless networks and mobile computing: 1-25.

[4] Perkins; Charles E. Ad hoc networking. Vol. 1. Reading: Addison-wesley, 2001.

[5] Zheng; S.; Wu; W.; Zhang; Q.; 2011, Energy and link-state based routing protocol for MANET, IEICE TRANSACTIONS on Information and Systems, 94 (5), 1026-1034.

[6] Tarique; Mohammed; Kemal E. Tepe; Sasan Adibi; Shervin Erfani; Survey of multipath routing protocols for mobile ad hoc networks, 2009, Journal of Network and computer applications 32, no. 6: 1125-1143.

[7] Jabbar: Waheb A.; Mahamod Ismail; Rosdiadee Nordin; Suki Arif; Power-efficient routing schemes for MANETs: a survey and open issues, 2017 Wireless Networks 23, no. 6: 1917-1952.

[8] Lutimath; Nagaraj M.; L. Suresh; Chandrakant Naikodi; Energy Aware Multipath AODV Routing Protocol for Mobile Ad hoc Network. 2016 International Journal of Engineering Research ISSN: 2319-6890.

[9] Aye; May Cho; Aye Moe Aung; Energy efficient multipath routing protocol for wireless mobile ad hoc networks. 2014 International Conference on Advances in Engineering and Technology.

[10] Omar; Smail; Mekkakia Zoulikha; Bernard Cousin; Energy efficiency in ad hoc wireless networks with node-disjoint path routing, 2011 In International Workshop on Systems, Signal Processing and their Applications, WOSSPA, pp. 127-130. 
[11] Sharma; Dhirendra Kumar; Amar Nath Patra; Chiranjeev Kumar; An update based energy-efficient reactive routing protocol for mobile ad hoc networks, 2013 International Journal of Computer Network and Information Security 5, no. 11: 17 .

[12] Chang; J. H; Tassiulas; L.; 2000, March., Energy conserving routing in wireless ad-hoc networks. In Proceedings IEEE INFOCOM 2000. Conference on Computer Communications. Nineteenth Annual Joint Conference of the IEEE Computer and Communications Societies (Cat. No. 00CH37064) (Vol. 1, pp. 22-31). IEEE.

[13] Agarwal; R.; Motwani; D.; 2009, Survey of clustering algorithms for MANET. arXiv preprint arXiv: 0912.2303.

[14] Smail; O.; Cousin; B.; Mekkakia, Z.; Mekki, R., 2014, November. Energy aware and stable Multipath Routing protocol in clustered wireless ad hoc networks. In 2014 IEEE/ACS 11th International Conference on Computer Systems and Applications (AICCSA) (pp. 222-229). IEEE.

[15] Agarwal; R.; Gupta; R.; Motwani; M.; 2012, REVIEW OF WEIGHTED CLUSTERING ALGORITHMS FOR MOBILE $\mathrm{AD}$ HOC NETWORKS. Computer Science \& Telecommunications, 33 (1).

[16] Preetha; V; Chitra; K.; 2015, Parameter Analysis for Clustering in Manet in Disaster Scenarios. In the 7th International Conference on Information Technology, ICIT 2015.

[17] Kanakala; S.; Ananthula; V. R.; Vempaty, P.; 2014, Energyefficient cluster based routing protocol in mobile ad hoc networks using network coding. Journal of Computer Networks and Communications, 2014.

[18] Basurra; S. S.; De Vos; M.; Padget; J.; Ji, Y.; Lewis, T.; Armour, S.; 2015, Energy efficient zone based routing protocol for MANETs. Ad Hoc Networks, 25, pp. 16-37.

[19] Zheng; S.; Wu, W.; Zhang, Q; 2011, Energy and link-state based routing protocol for MANET. IEICE TRANSACTIONS on Information and Systems, 94 (5), 1026-1034.

[20] Marina; M. K.; Das; S. R.; 2006, Ad hoc on - demand multipath distance vector routing. Wireless communications and mobile computing, 6 (7), pp. 969-988.

[21] Tarique, M.; Tepe; K. E.; Adibi; S.; Erfani; S.; 2009, Survey of multipath routing protocols for mobile ad hoc networks. Journal of Network and computer applications, 32 (6), pp. 1125-1143.

[22] Tarique; M.; Tepe; K. E.; Adibi; S.; Erfani; S; 2009, Survey of multipath routing protocols for mobile ad hoc networks. Journal of Network and computer applications, 32 (6), 11251143 .

[23] Kumar; R.; Dave; M.; 2011, A comparative study of Various Routing Protocols in VANET. arXiv preprint arXiv: 1108.2094.

[24] Sahu, R.; Sharma, S.; Rizvi, M. A.; 2019, ZBLE: Zone Based Leader Election Energy Constrained AOMDV Routing Protocol.

[25] Aye, M. C.; Aung, A. M.; 2014. A Modified Energy Constrained Protocol based on AOMDV for Mobile Ad-Hoc Networks. International Journal of Scientific Engineering and Technology Research, 3.
[26] Smail, O.; Cousin, B.; Mekki, R; Mekkakia, Z., 2014, A multipath energy-conserving routing protocol for wireless ad hoc networks lifetime improvement. EURASIP Journal on Wireless Communications and Networking, 2014 (1), p. 139.

[27] Manohari, P. K.; Ray, N. K.; 2015, October, EAOMDV: An energy efficient multipath routing protocol for MANET. In 2015 IEEE Power, Communication and Information Technology Conference (PCITC) (pp. 710-715). IEEE.

[28] Khan, K.; Goodridge, W., 2015, Energy aware Ad-Hoc on demand multipath distance vector routing. International Journal of Intelligent Systems and Applications, 7 (7), pp. 5056.

[29] Zarrad, A.; Alsmadi, I.; 2017, Evaluating network test scenarios for network simulators systems. International Journal of Distributed Sensor Networks, 13 (10), p. 1550147717738216.

[30] Ayele; E. D.; 2011, Analyses and deployement of the BitTorrent protocol for community ad hoc networks.

[31] Fall, K.; Varadhan, K.; 2005, The ns Manual (formerly ns Notes and Documentation). The VINT project, 47, pp. 19-231.

[32] Feeney; L. M.; Nilsson; M; 2001, April, Investigating the energy consumption of a wireless network interface in an ad hoc networking environment. In Proceedings IEEE INFOCOM 2001. Conference on Computer Communications. Twentieth Annual Joint Conference of the IEEE Computer and Communications Society (Cat. No. 01CH37213) (Vol. 3, pp. 1548-1557). IEEE.

[33] Huang; H.; Xu, Y.; Sun, Y. E.; Huang, L.; 2008, June, Cluster-based load balancing multi-path routing protocol in wireless sensor networks. In 2008 7th World Congress on Intelligent Control and Automation (pp. 6692-6696). IEEE.

[34] Yoon, J.; Liu, M.; Noble, B.; 2003, March, Random waypoint considered harmful. In IEEE INFOCOM 2003. Twentysecond Annual Joint Conference of the IEEE Computer and Communications Societies (IEEE Cat. No. 03CH37428) (Vol. 2, pp. 1312-1321). IEEE.

[35] Cano, J. C.; Manzoni, P; 2000, August, A performance comparison of energy consumption for mobile ad hoc network routing protocols. In Proceedings 8th International Symposium on Modeling, Analysis and Simulation of Computer and Telecommunication Systems (Cat. No. PR00728) (pp. 57-64). IEEE.

[36] Robbins; A. D.; 1996, E ective AWK Programming. Specialized Systems Consultants, Inc (SSC) and Free Software Foundation, 1 (3).

[37] Mokbal; F. M. M.; Saeed, K.; Dan, W.; 2018, Energy Consumption Evaluation of AODV and AOMDV Routing Protocols in Mobile Ad-Hoc Networks. Energy, 9 (8).

[38] Kulkarni; S. B.; Yuvaraju, B. N.; 2015, June, Node connectivity, energy and bandwidth aware clustering routing algorithm for real-time traffic multicasting in MANET. In 2015 IEEE International Advance Computing Conference (IACC) (pp. 760-763). IEEE.

[39] Fotino, M.; De Rango; F.; 2011, Energy issues and energy aware routing in wireless ad hoc networks (pp. 281-296). INTECH Open Access Publisher. 